\title{
Cool sensing
}

Superconducting quantum interference devices can accurately measure temperatures even below $1 \mathrm{mK}$, but there's more to them - as Thomas Schurig explains.

V isitors to the Physikalisch-Technische Bundesanstalt (PTB), Germany's national metrology institute, are always impressed by the huge cubic building (pictured) that houses the Berlin Magnetically Shielded Room 2 (BMSR2). Located in the very centre of Berlin, this area is not somewhere you would expect to find scientists performing precise magnetic measurements using extremely sensitive superconducting quantum interference device (SQUID) magnetometers. The 304-channel SQUID magnetometer system inside the BMSR2 is primarily used for biomedical investigations, material characterization and fundamental science, and senses a static background field of less than $500 \mathrm{pT}$, which is about a factor of $10^{5}$ weaker than the magnetic field of the Earth. This facility demonstrates the huge efforts required to exploit the full potential of state-of-the-art SQUID magnetometers in reaching sensitivities of a few fT.

SQUIDs consist of a superconducting loop interrupted by one (radio-frequency SQUID) or two (direct-current SQUID) Josephson junctions and are extremely sensitive devices, enabling the detection of weak magnetic signals, and small currents and voltages. Unsurprisingly, in the aftermath of their invention, by Robert Jaklevic and colleagues at the Ford Research Labs in the mid-1960s ${ }^{1,2}$, SQUIDs became attractive tools for standard laboratories. However, due to the lack of appropriate thin-film technologies for the fabrication of Josephson junctions, the first practical laboratory SQUIDs were equipped with mechanical point contacts consisting of a niobium screw and a niobium counterpart. Adjusting these contacts was a rather tricky task.

One of the first applications of SQUIDs in metrology was in low-temperature thermometry. At the beginning of the 1970s, Robert Kamper and James Zimmermann developed a primary temperature standard with point contact SQUIDs at the National Bureau of Standards in Boulder, Colorado. In their SQUID noise thermometer, the voltagenoise-generating resistor was an integral part of a so-called resistive SQUID (RSQUID) ${ }^{3}$. When accurate temperature measurements down to below $1 \mathrm{mK}$ were achieved with

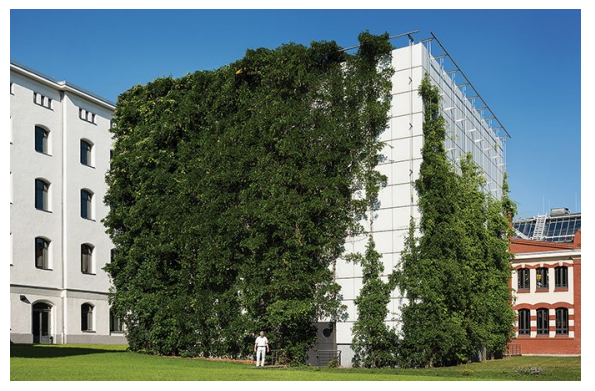

Credit: Physikalisch-Technische Bundesanstalt

RSQUIDs at PTB, a measuring time of about 15 hours was required to reach a statistical uncertainty of $10^{-3}$. RSQUID measurements played an important role in extending the International Temperature Scale ITS-90 to lower temperatures and in establishing the Provisional Low Temperature Scale PLTS-2000. Recently, more practical SQUID noise thermometers with state-of-the-art thin-film SQUIDs have been developed - among them a sophisticated primary magnetic-field-fluctuation thermometer, in which the SQUID inductively measures noise currents in a bulk copper sensor.

Apart from thermometry, SQUIDs are effectively used in electrical metrology. In 1972, Ian Harvey invented the so-called cryogenic current comparator (CCC), a superconducting transformer in which a SQUID acts as a null detector ${ }^{4}$. Key to electrical quantum metrology, this instrument enables measurements of resistance ratios or electric current ratios with the uncertainty down to about $10^{-9}$. Today, state-of-the-art CCC-based resistance bridges have become standard tools for relating the measurements of resistors to the quantum Hall resistance standard, ensuring that they are properly calibrated.

Since the 1990s, considerable effort has been spent on the development of SQUID current sensors, in which the current to be measured is fed into an input coil that is inductively coupled to a SQUID loop or array. These devices open up a wide variety of practical applications, including many in metrology. In particular, SQUID current sensors enable cryogenic radiation detection over a broad spectral range. Energy-dispersive $\mathrm{X}$-ray spectroscopy for chemical analysis, radionuclide spectroscopy and infrared singlephoton detection for characterizing quantum light sources are prominent examples of applications in metrology institutes ${ }^{5}$.

Despite these successes, challenges remain for SQUID measurement tools in metrology labs. In comparison to semiconductor devices, the standardization of superconducting electronic devices is still underdeveloped. Since 2005, under the auspices of the Institute of Electrical and Electronics Engineers, international joint efforts have been put into overcoming this obstacle.

The story of the SQUID is not finished yet. Nanotechnology and novel material combinations for Josephson junctions have enabled the fabrication of nanoSQUIDs intended for single-spin detection and magnetic microscopy. By pushing the limits of conventional technology, magnetic field noise levels of $150 \mathrm{aTHz}^{-1 / 2}$ have recently been achieved with SQUID magnetometers ${ }^{6}$ in PTB's magnetically shielded room BMSR2; subsequently improved magnetic shielding and auxiliary cryogenic components have been demanded. For this reason, PTB's BMSR2 is currently being remodelled with an additional inner permalloy layer to ensure that scientists can achieve even lower noise levels and higher sensitivity in magnetic measurements.

\section{Thomas Schurig}

Department Cryosensors, Physikalisch-Technische Bundesanstalt, Berlin, Germany.

e-mail: thomas.schurig@ptb.de

Published online: 1 August 2019

https://doi.org/10.1038/s41567-019-0604-7

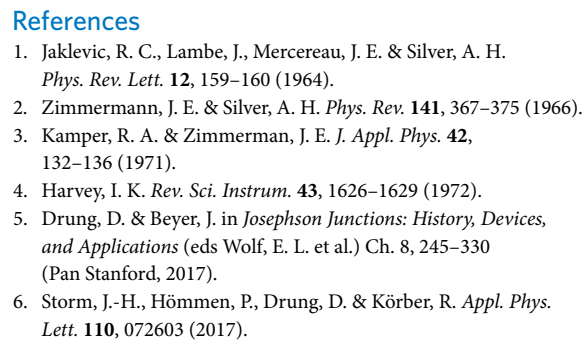

\section{ᄀ}

$\mathrm{m} e \mathrm{~s} \mathrm{\textrm {u }} R \mathrm{E}_{\mathrm{h}}$

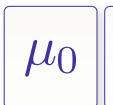

\section{$\varepsilon_{0}$}
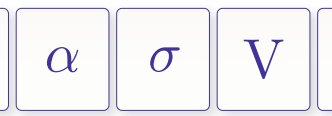

$\mathrm{R}$ 